\title{
L'informel et le non-marchand dans les systèmes d'activités : enquête représentative sur les ménages kanak en tribus de Nouvelle-Calédonie
}

\author{
Leïla Apithy ${ }^{1, *}$, Stéphane Guyard ${ }^{2}$, Séverine Bouard ${ }^{1}$, Michel Passouant ${ }^{3,5}$, Jean-Michel Sourisseau ${ }^{4,5}$ \\ et Jean-François Bélières ${ }^{4,5}$ \\ ${ }^{1}$ IAC, Nouvelles ruralités et destin commun, BP 0698825 Pouembout, Nouvelle-Calédonie \\ 2 IMT Atlantique, Dép. de sciences sociales et de gestion, CENS UMR 6025, 44307 Nantes, France \\ ${ }^{3}$ CIRAD, UMR TETIS, 34398 Montpellier, France \\ ${ }^{4}$ CIRAD, UMR ART-DEV, 34398 Montpellier, France \\ ${ }^{5}$ Univ Montpellier, Montpellier, France
}

\begin{abstract}
Résumé - Afin d'appréhender le rôle essentiel du travail de la terre et des activités de prélèvement (pêche et chasse) dans l'économie des tribus en Nouvelle-Calédonie, une enquête représentative de l'ensemble du pays, jusqu'à un niveau de 10 unités territoriales au sein des 3 provinces, a été réalisée en 2011 sur les activités de l'année 2010. Une partie des données de cette enquête est accessible au lien suivant: https:// dataverse.cirad.fr (DOI : 10.18167/DVN1/VWWVXU). Afin de permettre leur réutilisation, le présent article détaille le cadre conceptuel de l'enquête, la description du jeu de données, ainsi qu'une appréciation de sa qualité.
\end{abstract}

Mots clés : Nouvelle-Calédonie / tribu / agriculture / non-marchand / data paper

Abstract - Measuring informal and non-market activities: a survey representative for kanak households in tribes of New Caledonia. In 2011, a survey has been led to approach the role of agricultural activities (agriculture, breeding, fishing, hunting) in the economy of kanak households living in tribes in New Caledonia. The survey focuses on the activities held during the year 2010 and is representative of the population of the tribes at the country level as well as in 10 areas within the 3 provinces of New Caledonia. A set of the data collected is available at the following link: https://dataverse.cirad.fr (DOI: $10.18167 / \mathrm{DVN} 1 / \mathrm{VWWVXU}$ ). In order to allow people to use them for further analysis, this paper describes the conceptual framework, the nature and quality of the data.

Keywords: New Caledonia / tribe / agriculture / non-market / data paper

\section{Introduction}

Le présent article est un data paper (Dedieu, 2014). Les données présentées ici proviennent d'une enquête réalisée en Nouvelle-Calédonie auprès de groupes domestiques résidant en tribu (regroupements d'unités familiales issus de la colonisation). L'enquête réalisée en 2011 porte sur les activités de l'année 2010. Pour compléter l'article, les lecteurs trouveront dans les diverses publications produites dans le cadre de l'étude, les éléments de méthode et les

\footnotetext{
$\overline{\text { *Auteur de correspondance }}:$ leila.apithy@gmail.com
}

résultats obtenus (Apithy et al., 2014; Guyard et Apithy, 2014; Guyard et al., 2014). Par ailleurs, pour permettre la réutilisation des données, et devant la difficulté à interpréter des données sorties de leur contexte (Borgman, 2012), nous leur avons attaché une description détaillée par des métadonnées, selon la norme DDI (DDI Alliance, 2014). Pour la diffusion, un fichier unique au format Nesstar (Norwegian Centre for Research Data, 2017) regroupe métadonnées et données. Si le format Nesstar permet de diffuser données et métadonnées ensemble, l'exploitation effective des données elles-mêmes sera possible après exportation vers l'un des différents formats proposés (Stata, SPSS, csv pour Microsoft Excel...). 


\section{Contexte de l'étude}

La Nouvelle-Calédonie présente des modes d'organisation sociale rurale originaux (Djama, 1999), méconnus des systèmes officiels d'observation de l'agriculture. Le système statistique public de Nouvelle-Calédonie, compétence territoriale sous la responsabilité de l'Institut de la statistique et des études économiques (ISEE), reste donc fortement inspiré des modèles français métropolitains. La petite taille des unités de production, les modalités d'organisation domestique de la production et les orientations non marchandes pour la valorisation des produits par les familles, typiques des modèles agricoles kanak, sortent des standards classiquement observés. Pour certains acteurs du développement rural, la contribution de l'exploitation des ressources naturelles par les ménages vivant en tribu à la richesse du territoire était sous-évaluée.

Ainsi, à la demande de collectivités territoriales du pays - province Nord, province des Iles Loyauté et Gouvernement, tous trois copropriétaires des données -, l'Institut agronomique néocalédonien (IAC) a réalisé en 2011 une enquête pour évaluer la contribution des familles résidant en tribu à la production agricole, à l'élevage, à la pêche et à la chasse, ainsi que le rôle économique et social de ces productions pour ces familles.

Les données associées à cet article proviennent de cette enquête qui avait vocation à produire des statistiques valides pour l'ensemble de la Nouvelle-Calédonie, pour les provinces et sur de grandes «régions» infra-provinciales. L'enquête visait aussi à mieux comprendre les systèmes d'activités mis en œuvre par les familles résidant en tribu et la place des dimensions marchande et non marchande, matérielle et immatérielle. Elle entendait enfin identifier des leviers possibles, en rapport avec cette organisation sociale spécifique, pour renouveler les politiques de développement local. L'ensemble de l'étude, de par son caractère innovant dans la statistique calédonienne, relève d'une co-construction entre les commanditaires politiques et techniques et l'institution de recherche appliquée la mettant concrètement en œuvre.

\section{Cadre conceptuel}

Il s'agit d'appréhender le rôle essentiel du travail de la terre et des activités de prélèvement (pêche et chasse) dans l'économie des tribus (Bensa et Freyss, 1994; Leblic, 1989 ; Bouard, 2013 ; Gaillard et Sourisseau, 2009; Sourisseau et al., 2010).

Le choix de l'unité d'observation a été adapté au contexte particulier d'une organisation sociale coutumière, d'une agriculture familiale non contractuelle et dont la dimension non marchande prévaut. La démarche croise une approche par des unités d'observation stabilisées, renvoyant à des dispositifs d'enquête existant (ici le Recensement général de la population, RGP), et une approche compréhensive et qualitative repensant ces unités à la lumière de leur pertinence concrète et observée (Couty, 1983; Winter, 1983). Nous nous sommes ainsi inspirés de la notion d'unités socio-économiques, entités collectives exprimant les fonctionnalités de résidence, de consommation, de production agricole et d'accumulation (Gastellu, 1980). Ainsi, l'unité d'observation principale retenue est le «groupe domestique », constitué par l'ensemble des personnes résidant sur une même parcelle d'habitation, partageant les repas et une partie des travaux agricoles. Sont considérés comme appartenant à un groupe domestique les membres résidant à l'année dans l'une des habitations du groupe, mais également ceux habitant ailleurs la semaine (pour travailler, étudier...) mais rejoignant régulièrement le foyer en périodes de congés, ainsi que tout membre ayant passé au moins 6 mois dans le foyer au cours de l'année 2010. Le groupe domestique constitue également l'unité de base de l'accumulation, bien que celle-ci s'inscrive aussi dans des groupes plus englobants: clan et tribu, pour la terre notamment.

La population statistique concernée recouvre l'ensemble des groupes domestiques résidant en tribu, que les individus aient pratiqué une activité agricole ou non. Seul le critère géographique de résidence est déterminant.

Pour construire des modèles explicatifs du revenu global afin d'y saisir la place des activités rurales, une approche systémique a été retenue. Les caractéristiques démographiques, la conjugaison des différentes activités pratiquées, l'insertion dans des réseaux sociaux et institutionnels contribuent à rendre compte de l'organisation sociale des tribus. Les descriptions du cadre de vie et du niveau d'équipement des groupes domestiques participent à l'estimation de leur niveau de vie. Le type d'équipement agricole et le foncier mis en valeur renseignent sur la manière de produire. La prise en considération de l'ensemble des productions agricoles, qu'elles relèvent du secteur formel ou non, complétées par les autres activités, permet d'appréhender la richesse totale générée au sein des tribus ainsi que les rôles joués par ces produits dans l'entretien des liens sociaux.

En plus des informations relevant des enquêtes de structure (facteurs de production, système d'activités, capital humain, accès au marché, etc.) et du registre comptable agricole (volumes de production, charges, prix, marges nettes, etc.), les données collectées permettent de caractériser les différents indicateurs de capitaux mobilisés dans l'approche sustainable rural livelihoods (Chambers et Conway, 1991).

\section{Présentation du jeu de données}

Les données sont proposées dans un fichier au format Nesstar, intégrant aux données elles-mêmes l'ensemble des métadonnées au format DDI, et accessibles avec le logiciel Nesstar publisher librement téléchargeable.

\section{1 Échantillonnage et extrapolation}

Si l'unité d'observation et d'analyse est le groupe domestique, l'unité utilisée pour effectuer un échantillonnage aléatoire et représentatif est le ménage, au sens de l'ISEE (soit l'ensemble des individus résidant dans la même habitation principale; ainsi, un groupe domestique peut englober plusieurs ménages). L'échantillon initial a été tiré au sort dans la base des ménages résidant en tribu du RGP de 2009 et doublement stratifié selon des critères géographiques (10 zones infra-provinciales) et la taille du ménage ( 7 strates). Selon l'expertise et les pratiques de l'ISEE, un taux de sondage uniforme de $14 \%$ des ménages a été appliqué dans chacune des 70 strates. L'échantillon final diffère légèrement de ce plan de sondage et puise pour partie hors des listes du recensement. $\mathrm{Au}$ 
Tableau 1. Principaux thèmes explorés et dénombrement des lignes et des variables.

Table 1. Main thematics and number of individuals and variables.

\begin{tabular}{|c|c|c|c|c|}
\hline Principaux thèmes & $\begin{array}{l}\text { Unité } \\
\text { d'observation }\end{array}$ & $\begin{array}{l}\text { Nombre } \\
\text { de lignes }\end{array}$ & Principaux sous-thèmes & $\begin{array}{l}\text { Nombre de } \\
\text { variables }\end{array}$ \\
\hline Ensemble & - & - & & 136 \\
\hline Démographie & Individu & 6652 & $\begin{array}{l}\text { Âge, genre, place dans le groupe domestique } \\
\text { Scolarité } \\
\text { Activités pratiquées et temps de travail }\end{array}$ & 15 \\
\hline Équipement et foncier & GD & 1429 & $\begin{array}{l}\text { Équipement } \\
\text { Équipement agricole } \\
\text { Foncier utilisé }\end{array}$ & 18 \\
\hline Volumes de production & GD & 1429 & $\begin{array}{l}\text { Volumes de production agricole et prélèvement (chasse, pêche) } \\
\text { Destination des produits }\end{array}$ & 50 \\
\hline
\end{tabular}

$\mathrm{GD}=$ groupe domestique.

final, 1786 ménages ont été interrogés, ce qui correspond à $12,5 \%$ des ménages de tribu. Les groupes domestiques ont été reconstitués au moment de l'enquête en examinant les liens existants avec d'autres ménages et en particulier la prise en commun des repas.

L'extension des analyses à l'ensemble de la population se fait en utilisant des estimateurs d'Horvitz-Thomson (Ardilly, 2006). À cet effet, un coefficient d'extrapolation a été calculé pour chaque groupe domestique. Le calcul a été effectué en trois étapes : le redressement de l'échantillon des ménages, le calcul d'un coefficient au niveau du groupe domestique comme une moyenne des coefficients des ménages le composant, pondérée par le nombre de membres de chacun d'eux, et enfin un arrondi du coefficient ainsi obtenu. Le biais d'échantillonnage a été calculé en rapprochant les effectifs de la population totale recensée de ceux mesurés avec nos estimations, ce qui a permis de valider à la fois l'échantillon, les coefficients et les estimateurs choisis.

\subsection{Description des données}

Les données portent sur 1429 groupes domestiques. Elles sont organisées en 4 tableaux construits au niveau des groupes domestiques (1429 lignes) ou détaillés au niveau de chacun de leurs membres (6652 lignes).

Le Tableau 1 présente l'organisation des principaux thèmes, avec le nombre de lignes et de variables afférentes, pour une présentation synthétique développée par la suite. Le détail du dictionnaire de données est accessible dans le fichier Nesstar: nom des variables, définition, format, nature, paramètres de statistiques descriptives...

Les variables sont de trois types:

- quantitatives discrètes, les effectifs sont exprimés en nombres entiers ;

- quantitatives continues, exprimées sous forme de nombres décimaux, et dont les unités sont des ares pour les superficies, des francs Pacifique (FCFP) pour les quantités monétaires ;

- qualitatives, exprimées en caractères, avec le libellé explicite de la modalité concernée.

\subsubsection{Identification}

Dans tous les tableaux de données, un groupe de variables d'identification, mais préservant l'anonymat:

- identifie chaque ligne des tableaux et la resitue de manière géographique, que ce soit pour un groupe domestique ou un ménage avec ses membres. Il faut prendre garde au fait que les codes de groupe domestique sont particuliers à chaque tableau et ne sauraient être utilisés pour effectuer des jointures entre tableaux;

- précise les coefficients d'extrapolations, calculés pour chaque groupe domestique après redressement de l'échantillon. Pour le fichier individuel comme pour ceux relevant du groupe domestique, la pondération doit être appliquée à chaque ligne pour produire des résultats représentatifs de l'ensemble de la population des tribus.

\subsubsection{Démographie}

Ces données, au niveau individuel, caractérisent la population des tribus et renseignent sur la composition des groupes domestiques et des ménages. Pour chaque membre sont précisés l'âge, le sexe, la relation avec le chef de groupe et de ménage, le niveau scolaire et le plus haut diplôme obtenu, ainsi que des informations sur les activités génératrices de revenu pratiquées au cours de l'année 2010 et le temps consacré à chacune d'elles.

\subsection{3 Équipement et foncier}

L'équipement des groupes domestiques est décrit par le mode d'accès à l'eau et à l'électricité, la possession de téléphone portable et de réfrigérateur. 
Le matériel agricole a été classé en quatre catégories : le petit matériel (sabres d'abattis, barres à mine, pioches etc.); le matériel intermédiaire (motoculteurs, pulvérisateurs à main, débroussailleuses, etc.); les gros équipements motorisés (tracteurs, attelages et engins de chantiers utilisés pour l'agriculture); et enfin le matériel «spécialisé », spécifique à une activité et peu répandu (four à coprah, matériel d'apiculture, etc.).

Concernant le foncier, l'ampleur du disponible, en opposition à la petite taille des parcelles cultivées, nous a conduit à ne prendre en compte que le foncier effectivement mis en valeur au cours de l'année 2010. Les données font ainsi état de la surface des parcelles cultivées avec des plantes annuelles ou pérennes (vergers, cocoteraies, etc.).

Les surfaces ont été estimées par les enquêteurs quand il leur était possible d'accéder aux parcelles ou à partir de la déclaration des répondants. Dans tous les cas, les données ont fait l'objet d'un apurement et cette variable a, au besoin, été réestimée sur la base du type de cultures plantées, du nombre de plants ou d'arbres et de leur emprise au sol.

\subsubsection{Volumes de production}

Pour évaluer la contribution des tribus à la production agricole calédonienne, il était indispensable de déterminer les volumes de production (végétale et animale) et de prélèvement (chasse et pêche). Ils ont été reconstitués avec les personnes interrogées pour l'ensemble de l'année 2010. La grande majorité des groupes domestiques ne faisant pas de pesée de leurs récoltes, ne tenant pas de registre de comptabilité, le questionnaire prévoyait de s'appuyer sur les pratiques quotidiennes en prenant en compte la diversité des outils utilisés pour la récolte (sacs plastiques, cabas de courses, paniers en feuilles de cocotier, etc.) et la façon de récolter (à la semaine, au mois, au moment des coutumes). Ces quantités ont par la suite été converties dans les unités de mesure conventionnelles à l'aide d'abaques constitués pour l'occasion à partir des résultats de mesures sur un échantillon d'unités utilisées localement.

\subsubsection{Revenu}

Le revenu total du groupe domestique a été décomposé ainsi : les revenus liés aux activités de travail non agricole, les revenus sociaux, les revenus « exceptionnels » (dons, gains aux jeux, héritages, etc.) et les revenus liés aux activités agricoles et de prélèvement (RAP). Les trois premières composantes ont été estimées directement à partir de la déclaration des répondants, tandis que la dernière a fait l'objet d'un calcul après recueil de ses différents termes.

Les productions non commercialisées (conservées pour l'autoconsommation ou données au cours de cérémonies coutumières ou au quotidien) participent de la richesse totale créée par un groupe domestique. Afin d'estimer cette richesse non marchande, nous avons affecté le prix du marché aux productions ou prélèvements non commercialisés. Le RAP total correspond alors à la somme des recettes de la commercialisation et de la valorisation du non marchand, à laquelle sont retranchés les coûts de production annuels et les amortissements du matériel agricole et de pêche. En affectant le ratio Valorisation du non marchand / Recette de la commercialisation à ces deux dernières variables, il est alors possible de calculer un RAP marchand et un RAP non marchand, dont la somme correspond au RAP total.

\subsection{Couverture géographique}

L'enquête a été réalisée dans 288 des 340 tribus que compte la Nouvelle-Calédonie. Une fois extrapolées, les données sont représentatives des ménages des tribus à l'échelle de la Nouvelle-Calédonie, des trois provinces (Nord, Sud et Îles Loyauté), ainsi que de 10 zones infra-provinciales.

Afin de garantir l'anonymat des répondants, les données ne sont pas fournies à un niveau géographique inférieur à ces 10 zones (communes ou tribus).

\subsection{Couverture temporelle}

Les données collectées portent sur l'ensemble de l'année 2010. Les volumes horaires de travail et les volumes de production ont été totalisés pour l'ensemble de l'année 2010. Un individu a été considéré comme actif dès lors qu'il prenait part à une activité procurant des ressources ou des revenus, même si cette activité pouvait être présentée comme un loisir (valorisation des produits de la pêche à travers l'autoconsommation ou l'échange, par exemple), et même si elle n'avait été pratiquée que ponctuellement et ne l'était plus au moment de l'enquête. Les individus de moins de 15 ans n'ont pas été comptés parmi les actifs, même s'ils participaient aux productions agricoles ou aux prélèvements; le produit de leur travail a cependant été pris en compte dans le total du groupe domestique.

\section{5 Éléments de qualité}

Le détail des choix méthodologiques effectués pour conduire cette enquête a fait l'objet d'une publication (Apithy et al., 2016b), partiellement reprise dans les lignes qui suivent, ainsi que d'un document de travail (Apithy et al., 2014).

\subsection{Dispositif d'enquête}

Le recueil des informations a été réalisé avec un questionnaire administré en face à face pour, en un seul passage, collecter l'ensemble des données. Ce questionnaire est joint aux métadonnées. Son élaboration a mobilisé de nombreuses personnes ressources, depuis la formulation des questions jusqu'aux spécifications des unités de mesure ou au contenu des nomenclatures à utiliser. Le questionnaire a été validé après plusieurs tests de terrain.

L'équipe en charge de la collecte sur le terrain était composée de deux coordinateurs, deux superviseures de terrain, dix enquêteurs et trois stagiaires.

Afin d'assurer le meilleur accueil et la meilleure disponibilité possible de la part des personnes interrogées, l'étude avait été présentée aux autorités coutumières et un courrier individuel envoyé aux groupes domestiques préalablement au passage des enquêteurs. De manière générale, l'enquête a été bien accueillie avec des entretiens qui duraient en moyenne une heure et demie. 
Les données collectées ont ensuite été informatisées via le logiciel Microsoft Access@

\subsection{Qualité de l'échantillonnage}

L'échantillon initial tiré par l'ISEE était constitué de 2000 ménages, complété par une liste de remplacement de 2000 autres noms, afin d'anticiper les absents ou les refus de répondre. Malgré cela, les enquêteurs ont dû faire face à un nombre important d'absents, qui ont dû être remplacés par des ménages «disponibles» choisis au hasard dans la localité.

Cette adaptation n'a pas de conséquence sur la représentativité finale des données ni sur leur qualité, le taux d'échantillonnage final et la valeur des coefficients d'extrapolation ayant été contrôlés et redressés pour chacune des strates avec un retour sur le terrain et un supplément de collecte si besoin était.

Sur l'objectif initial large de 2000 ménages, le taux de réalisation effectif (questionnaires remplis intégralement après contrôle et complément au téléphone si besoin) est de $89 \%$, soit un échantillon final de plus de $12,5 \%$ des ménages résidant en tribu.

Pour contrôler la qualité de l'échantillon et des redressements effectués, nous avons utilisé les estimateurs permettant de recalculer le nombre de personnes recensées dans la base de sondage. Il en résulte, sur l'ensemble du territoire, une très légère sous-estimation de la population à partir des données issues de l'enquête (Tab. 2).

\subsection{Qualité des données}

Les données ont fait l'objet d'un travail d'apurement avec correction des erreurs de saisie et des incohérences, contrôle des valeurs extrêmes et des incomplétudes, et recherche des doublons (Berti-Equille, 2006). Des traitements des données brutes ont permis de produire les fichiers à analyser.

Les corrections opérées par l'équipe d'enquête, parfois avec appel téléphonique, voire retour sur le terrain, ont permis la constitution d'un jeu de données sans manque et considéré sans erreur, dont la qualité est corroborée par la concordance avec les résultats issus d'autres dispositifs d'enquête (ISEE, 2010; DAVAR, 2011; ISEE, 2015).

Le jeu de données que nous proposons ici est une extraction sélective du jeu original. Il contient à la fois des données brutes, directement issues de la collecte, ainsi que le résultat de traitements (agrégation des unités secondaires au niveau du groupe domestique et certains calculs comme, par exemple, le RAP).

\subsection{Anonymisation}

Afin de garantir l'anonymat des répondants, les noms, prénoms, adresses et numéros de téléphone ne font pas partie des données fournies. Le niveau de localisation géographique le plus fin est celui de la zone infra-provinciale, aucune donnée ne relève du niveau communal ou de la tribu. Enfin, dans l'éventualité où, malgré ces précautions, certains groupes domestiques pourraient être identifiés, les données ont été organisées en 4 tables thématiques indépendantes et l'ordre des enregistrements ainsi que les identifiants des groupes
Tableau 2. Évaluation de la qualité de l'échantillon et des redressements.

Table 2. Sample and adjustment assessment.

\begin{tabular}{llll}
\hline & $\begin{array}{l}\text { Population } \\
\text { extrapolée sur } \\
\text { l'échantillon }\end{array}$ & $\begin{array}{l}\text { Population } \\
\text { recensée } \\
\text { ISEE }\end{array}$ & $\begin{array}{l}\text { Écart en } \\
\text { pourcentage } \\
\text { enquête/ISEE }\end{array}$ \\
\hline Ensemble & 56662 & 56995 & $-0.58 \%$ \\
\hline
\end{tabular}

domestiques diffèrent d'une table à l'autre. Chacune de ces tables a été étudiée avec des experts du secteur agricole calédonien et aucune variable pseudo-identifiante n'a été repérée. Cependant, le cas échéant, seules les informations présentes dans la même table thématique seraient révélées ; le lien ne pourrait pas être fait pour l'ensemble des autres informations portant sur ce groupe domestique. $\mathrm{Si}$ des utilisateurs souhaitent croiser les données des différentes tables, des conditions d'accès spécifiques pourront être discutées et encadrées par l'IAC.

\subsection{Métadonnées}

Les métadonnées jointes aux tableaux de données sont organisées, selon la norme DDI mise en oeuvre avec Nesstar Publisher, en 5 grandes sections (Fig. 1):

- la description du document (données et métadonnées) avec son titre, ses auteurs, producteurs et mode de diffusion, son mode de citation... Il s'agit, en quelque sorte, des métadonnées sur les métadonnées;

- la description de l'étude, avec des informations globales sur l'enquête : contenu des données, méthode, conduite de l'enquête, unités d'observation, couverture spatiale et temporelle, auteurs, producteurs, bailleurs, diffusion, conditions d'accès...;

- la description des tableaux, avec pour chaque table son format, ses dimensions, leurs traitements...;

- la description des variables de chaque tableau, avec leur type, leur nom et label associé (nom long), leur définition, la nomenclature des variables qualitatives...;

- les documents complémentaires (questionnaire, guide de l'enquêteur, plaquettes synthétiques des principaux résultats).

\section{Conclusion}

\subsection{Principaux résultats}

Les analyses produites ont permis :

- de faire une description fine de la population résidant en tribu;

- d'améliorer la connaissance sur les modes de fonctionnement de ces groupes sociaux;

- d'obtenir une évaluation du poids des activités agricoles et de prélèvement pour ces groupes domestiques, s'agissant du temps qui y est consacré, des volumes et des valeurs produits et de la place importante de la «richesse non monétaire» dans le revenu total des familles, et plus largement $\mathrm{du}$ pays. Cette démonstration $\mathrm{du}$ poids significatif des activités tribales dans la reproduction 


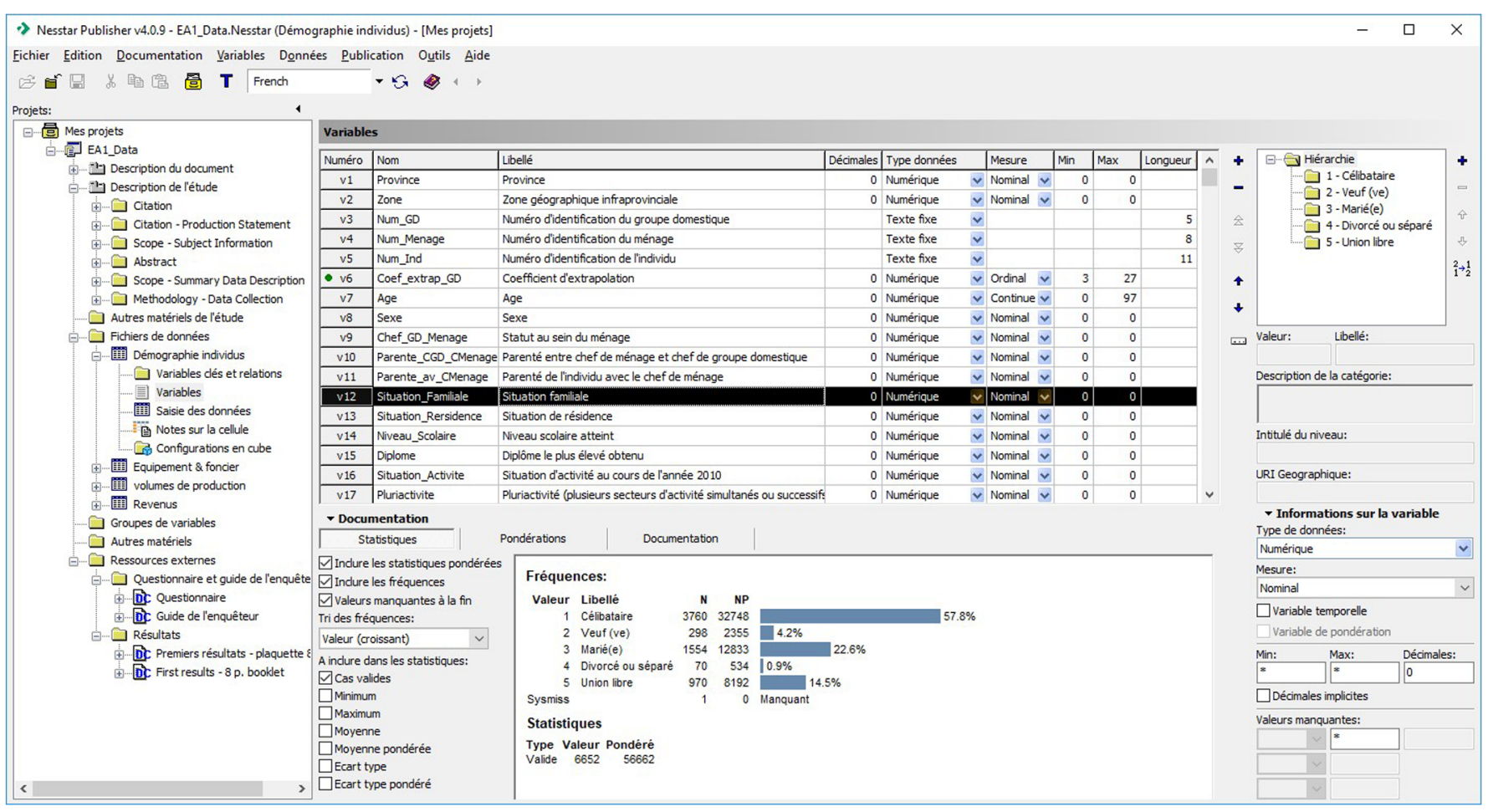

Fig. 1. Données et métadonnées sous Nesstar Publisher.

Fig. 1. Data and metadata on Nesstar publisher.

socio-économique des familles a permis de renouveler le débat sur les politiques de développement rural, notamment dans les refontes des codes de développement provinciaux.

L'analyse des données collectées a déjà donné lieu à la publication d'un rapport d'étude (Guyard et al., 2014) et de chapitres d'ouvrages (Bouard et al., 2015; Guyard et Apithy, 2014; Apithy et al., 2016a), qui proposent des analyses spécifiques.

\subsection{Perspectives}

Des analyses supplémentaires pourraient être effectuées, car les données disponibles sont très riches et pour certaines insuffisamment valorisées, notamment en ce qui concerne le fonctionnement du groupe domestique en lien avec l'importance des échanges et des revenus non monétaires. Des travaux d'analyse pourraient également être effectués en mettant en relation ces données et les données produites par les autres systèmes de collecte, afin d'obtenir une caractérisation plus complète du secteur agricole. De plus, des croisements avec des données relevant d'autres disciplines seraient intéressants ; ainsi, les données pourraient être rapprochées d'études portant sur l'environnement, la gestion de l'eau, l'alimentation, la santé, etc. Des comparaisons avec le fonctionnement des unités économiques, en lien avec les politiques publiques mises en place dans d'autres pays présentant des systèmes similaires, apporteraient également un éclairage intéressant.

Apportant une information chiffrée sur une population statistique mal connue et une thématique peu mesurée jusqu'à présent, ces données pourraient aider à la mise en place de nouvelles enquêtes en fournissant des éléments quantitatifs pour mieux dessiner les questionnaires et en calibrer l'échantillonnage (Bem et al., 2008).

Enfin, le caractère statique des données produites limite leur exploitation en termes de simulation et de prospective de la place des activités extractives dans les équilibres du pays. Une recommandation serait de reconduire régulièrement une telle enquête dans le temps, ce qui permettrait une exploitation dynamique en associant au besoin différentes équipes de recherche et de développement, notamment pour des travaux d'économétrie.

Ces différentes perspectives mettent en évidence l'intérêt de construire un observatoire pluridisciplinaire à l'échelle du pays. Un tel projet offrirait des pistes plus solides pour l'élaboration et la mise en œuvre de politiques publiques adaptées.

\subsection{Précautions}

Pour utiliser les données, il convient de s'imprégner du cadre conceptuel qui a prévalu à la définition des variables et des unités d'observation, en particulier les groupes domestiques.

Par ailleurs, les données concernent l'année 2010, et ne sont représentatives qu'après application des coefficients d'extrapolation et par zone géographique.

On rappelle que, pour préserver l'anonymat, les quatre tableaux de données sont indépendants. Bien que concernant les mêmes unités statistiques, ils ne sauraient être rapprochés car ils présentent des identifiants spécifiques et la distribution des enregistrements diffère de l'un à l'autre. Les données originales comportent plus de 70 tables liées. Leur utilisation 
nécessite une forte implication dans la logique de l'étude. Néanmoins, il est envisageable d'y accéder dans le cadre de conventions à passer avec l'IAC.

Les données sont mises à disposition selon les termes de la licence Creative Commons CC-BY-NC-SA-4.0: AttributionNonCommercial-ShareAlike 4.0 International. Elles ne devront en aucun cas être utilisées à des fins commerciales et pourront être repartagées dans les mêmes conditions que les originales.

Toute utilisation des données doit faire mention de leur source. Il convient de les citer comme suit:

Apithy Leïla; Guyard Stéphane; Bouard Séverine; Passouant Michel; Sourisseau Jean-Michel ; Bélières Jean-François, 2017, "Agriculture des tribus de Nouvelle-Calédonie" [Base de données], IAC, récupérée à partir de CIRAD Dataverse, https://dataverse.cirad.fr, DOI : 10.18167/DVN1/VWWVXU.

\section{Références}

Apithy L, Guyard S, Bouard S, Passouant M, Sourisseau J-M., Bélières J-F. 2014. Réaliser une enquête par sondage: une application au fonctionnement des unités socio-économiques du monde rural Kanak (Nouvelle-Calédonie). Document de travail ART-Dev 2014-16. Montpellier (France) : CIRAD. http://art-dev. cnrs.fr/IMG/pdf/wpARTDev_2014_16.pdf.

Apithy L, Bouard S, Gorohouna S, Guyard S, Sourisseau JM. 2016 a. Dynamiques économiques et sociales des tribus et de la ruralité : fragilités et facultés d'adaptation. In : Bouard S, Sourisseau J-M, Geronimi V, Blaise S, Ro'i L, eds. La Nouvelle-Calédonie face à son destin. Quel bilan à la veille de la consultation sur la pleine souveraineté?. Paris: Hommes et Sociétés, pp. 315-351.

Apithy L, Guyard S, Bouard S, Passouant M, Sourisseau J-M, Bélières JF. 2016b. Adapter les méthodes d'enquêtes à une agriculture principalement non marchande: un exemple en Nouvelle-Calédonie. Cahiers Agricultures 25: 35006. DOI: 10.1051/cagri/2016024.

Ardilly P. 2006. Les techniques de sondage. Paris: Technip.

Bem J, Mba M, Subran L. 2008. Calcul de précision et plan de sondage: application aux enquêtes camerounaises auprès des ménages (ECAM2 et ECAM2). STATECO 102: 14.

Bensa A, Freyss J. 1994. La société kanak est-elle soluble dans l'argent? Terrain Rev. Ethnol. Eur. (23): 11-26. DOI: 10.4000/ terrain.3098.

Berti-Equille L. 2006. Qualité des données. Techniques de l'ingénieur. Informatique HB4 (H3700).

Borgman CL. 2012. The conundrum of sharing research data. Journal of the Association for Information Science and Technology 63-6: 1059-1078.

Bouard S. 2013. La ruralité kanak à la recherche d'un modèle décolonisé. Culture Océanienne. Tahiti : Au vent des îles.

Bouard S, Apithy L, Guyard S. 2015. L'agriculture familiale dans la société kanak contemporaine. In : Bosc PM, Sourisseau JM, Bonnal P, Gasselin P, Valette E, Bélières JF, eds. Diversité des agricultures familiales. Versailles (France): QUAE, pp. 315-326.
Chambers R, Conway G. 1991. Sustainable rural livelihoods: practical concepts for the 21 st century. IDS Discussion Paper 296. Brighton: IDS.

Couty P. 1983. Systèmes et rapports de production. Recherches de l'ORSTOM sur les terroirs, les groupes ethniques et les régions d'Afrique noire. Qualitatif et quantitatif: Deux modes d'investigation complémentaires. Réflexions à partir des recherches de l'ORSTOM en milieu rural africain, notes de travail. AMIRA 43: 3-16.

DDI Alliance. 2014. Data Documentation Initiative. [2017/10/10]. http://www.ddialliance.org/.

Dedieu L. 2014. Rédiger et publier un data paper dans une revue scientifique, en 5 points. Montpellier : Cirad, 7 p. http://url.cirad.fr/ ist/data-paper.

Djama M. 1999. Transformations agraires et systèmes ruraux mélanésiens en grande terre de Nouvelle-Calédonie. J. Agric. Tradit. Bot. Appl. 41(1): 201-224.

DAVAR. 2011. Mémento agricole, données 2010. Nouméa: DAVAR, $7 \mathrm{sp}$.

Gaillard C, Sourisseau JM. 2009. Système de culture, système d'activité(s) et rural livelihood : enseignements issus d'une étude sur l'agriculture kanak (Nouvelle-Calédonie). J. Soc. Océan. 129: 279-294. DOI: 10.4000/jso.5889.

Gastellu JM. 1980. Mais, où sont donc ces unités économiques que nos amis cherchent tant en Afrique? Cah. ORSTOM, Sér. Sci. Hum. 17(1-2): 3-11.

Guyard S, Apithy L. 2014. L'arrière plan ethnologique d'une enquête quantitative. Réflexion à partir d'une enquête statistique sur les activités des ménages résidant en tribu en Nouvelle-Calédonie. In : Fillol V, Le Meur PY, eds. Terrains Océaniens: Enjeux et Méthodes. Paris: L'Harmattan, (Cahiers du Pacifique Sud contemporain ; $\mathrm{n}^{\circ}$ h.s. 2), pp. 251-266.

Guyard S, Apithy L, Bouard S, Sourisseau JM, Passouant M, Bosc PM, et al. 2014. L'agriculture des tribus en Nouvelle-Calédonie. Résultats d'une enquête de 2010 sur la place et les fonctions de l'agriculture, l'élevage, la pêche et la chasse pour les groupes domestiques résidant en tribu. Nouvelle-Calédonie: IAC/Cirad, 230 p. + Annexes.

ISEE. 2010. Enquête Budget, Consommation des ménages. Synthèse 18: $4 \mathrm{p}$.

ISEE. 2015. Tableaux de l'économie calédonienne, $133 \mathrm{p}$.

Leblic I. 1989. Les clans pêcheurs en Nouvelle Calédonie : le cas de l'île des Pins. Cah. Sci. Hum. 25(1-2): 109-123.

Norwegian Centre for Research Data, n., d. Nesstar Publisher. [2017/ 10/10]. http://www.nesstar.com/software/publisher.html.

Sourisseau JM, Pestaña G, Gaillard C, Bouard S, Mennesson T. 2010. À la recherche des politiques rurales en NouvelleCalédonie. Trajectoires des institutions et représentations locales des enjeux de développement (1853-2004). Nouméa: IAC Éditions.

Winter G. 1983. Deux méthodes d'investigation irréductibles mais complémentaires. Recherches de l'ORSTOM sur les terroirs, les groupes ethniques et les régions d'Afrique noire. Qualitatif et quantitatif: Deux modes d'investigation complémentaires. Réflexions à partir des recherches de l'ORSTOM en milieu rural africain, notes de travail. AMIRA 43: 78-89. 